\title{
Mass Psychogenic Illness: Psychological Predisposition and Iatrogenic Pseudo-vocal Cord Dysfunction and Pseudo-reactive Airways Disease Syndrome
}

\author{
Herman Staudenmayer • Kent L. Christopher • \\ Lawrence Repsher • Ronald H. Hill
}

Published online: 8 February 2011

(C) American College of Medical Toxicology 2011

\begin{abstract}
A multidisciplinary team assessed five patients who alleged chronic medically unexplained multiorgan system symptoms described by idiopathic environmental intolerance allegedly triggered by exposure to solvents used in membrane roofing repair work on an office building. The event precipitated an incident of mass psychogenic illness (MPI). Treating physicians diagnosed irritant-associated vocal cord dysfunction (IVCD) and reactive airways disease syndrome (RADS) resulting from exposure. The authors conducted medical, psychological, and industrial hygiene evaluations. Air monitoring data for total volatile organic compounds obtained during the 2-day exposure period, measurements of emissions during membrane roofing repair at a similar site, mathematical modeling of air contaminant concentrations, and injection of tracer gas into the incident building revealed exposure levels well below those doses anticipated to cause clinical symptoms. There was no objective medical evidence validating symptoms. Review of the medical records indicated that the video laryngoscopy data, pulmonary function tests, and medical examinations relied upon by the treating physicians were inconsistent with published criteria for IVCD and RADS. Psychological evaluation identified defensiveness and self-serving misrepresentations of exaggerated health
\end{abstract}

H. Staudenmayer $(\bowtie)$

Denver, CO, USA

e-mail: hstaudenmayer@msn.com

K. L. Christopher $\cdot$ L. Repsher

Associate Clinical Professor of Medicine,

University of Colorado Health Sciences Center,

Denver, CO, USA

R. H. Hill

Industrial Hygiene and Safety, Landmark Environmental, Inc.,

Denver, CO, USA concerns associated with somatization and malingering. Each case had personality traits associated with at least one personality disorder. Social histories identified premorbid life events and stressors associated with distress. This is the first study to assess psychological predisposition, social interaction among the plaintiffs, and iatrogenic reinforcement of beliefs by diagnoses of pseudo-disorders associated with patient misrepresentation of exaggerated health concerns in an incident of MPI.

Keywords Mass psychogenic illness (MPI) - Idiopathic environmental intolerance (IEI) - Irritant induced vocal cord dysfunction (IVCD) - Solvent · Toluene · Indoor air quality · Volatile organic compounds (VOC) - Reactive airways disease syndrome (RADS) - Somatoform disorder

Mass psychogenic illness (MPI) has manifested as medically unexplained symptoms modulated by cultural and social contexts since recorded history [1-3]. Today, MPI often involves odors, actual or perceived, believed to be toxic and originating from a specific site of exposure such as in "sick building syndrome" $[4,5]$. Low-level exposure to volatile organic compounds (VOCs) may trigger sensory perception and higher levels may produce transient symptoms, such as headache or symptoms that arise from direct mucosal irritation. However, no data can explain how disability from chronic severe multiorgan system symptoms occurs in the absence of exposure to levels sufficient to cause documented injury [6]. The financial and social burdens associated with MPI are significant [7].

On 3-4 November 1999, several office employees working on the third floor in a four-storied office building with approximately 250 employees in Denver, Colorado, reported experiencing ill-health effects. Air contaminants reportedly entered the rooftop outside air intake of the building's heating, 
ventilation, and air-conditioning (HVAC) system during installation of a new roofing membrane. Subsequently, five office workers reported chronic and debilitating ailments 3 years after this event. They had associated "diagnoses" of reactive airways dysfunction syndrome (RADS) [8], irritantassociated vocal cord dysfunction (IVCD) [9], and a wide spectrum of symptoms manifesting as intolerance to multiple low-dose chemicals characteristic of idiopathic environmental intolerance (IEI) [10].

This paper reports a detailed assessment of this episode addressing (1) establishing the exposure level in an incident of alleged hazardous chemical release; (2) validating patient symptoms by objective, standardized medical methodology; (3) assessing social and individual psychological factors that predispose certain individuals to initiate or to be affected by MPI epidemics; (4) identifying factors that maintain chronicity after removal from exposure; and (5) underscoring the role physicians play by asserting that the illness was a toxic response, thereby reinforcing false beliefs and adding an iatrogenic component to the individual's dysfunction.

\section{Methods}

Medical evaluations consisted of a thorough review of all available records, a detailed history of prior medical problems, a thorough physical examination with emphasis on relevant organ systems of concern, complete pulmonary functions tests, and a methacholine challenge test. Blood tests were conducted by treating physicians, but since these were respiratory cases, there was no indication for basic laboratory work and that any positive findings would have been irrelevant.

Psychological evaluations were conducted 3 years after the incident with written informed consent. Interview assessed mental status, symptoms and belief about causation, history of the exposure incident, a brief medical history, and social history. Current symptoms of distress were assessed by the Derogatis Symptom Checklist (SCL-90R) [11]. In the analysis, the two categories with the highest $\mathrm{T}$ scores based on a normative sample were selected. The Minnesota Multiphasic Personality Inventory, 2nd edition (MMPI-2)
[12] and the Millon Clinical Multiaxial Inventory, 2nd edition (MCMI-II) [13] were administered to assess psychopathology and personality traits. Two performance-based tests of effort were used to objectively measure motivation rather than ability or neurocognitive impairment; the computerized assessment of response bias (CARB) [14], and the word memory test (WMT) [15].

A detailed industrial hygiene evaluation was done to: (1) determine the materials and methods used to install the membrane roofing of the incident building; (2) create a model of the exposure measuring the air contaminant emissions from a membrane roofing activity at another building under similar conditions; (3) model air contaminant concentrations that may have occurred on 3-4 November 1999; (4) calculate the theoretical worst-case air contaminant concentration by assuming a chemical spill within the building; and (5) inject a tracer gas into the building's ventilation system to simulate potential roofing emissions intake with current measurements of indoor air concentrations.

\section{Results}

The demographics of the five cases are presented in Table 1 . They were mostly female (four of five), middle-aged (mean= 46.2 years), white-collar technicians with a high school education. They were life-long non-smokers. All were divorced at least once and remarried, and all had children.

\section{Incident History for Individual Cases}

Case no. 1 She was the index case, defined as the individual at the center of the reported incident. On 3 November 1999, she recalled experiencing symptoms of headache and dizziness in the morning. During the lunch period, she went outside and talked to others. Upon returning to the building, she also experienced cough (a symptom of one of the other patients with whom she met). At 2:15 p.m., the administrators announced that people could go home because of reported symptoms. The next morning, while working in the same area of the building, she recalled smelling fumes of glue and burning tar and had a metallic taste in her mouth. It should be noted

Table 1 Demographics

\begin{tabular}{llccllll}
\hline Case no. & Sex & Age (years) & Education (years) & Smoking history & Job description & Marital status & Number of children \\
\hline 1 Index & F & 42 & 12 & Never & Computer specialist & Divorced remarried & 3 \\
2 & F & 49 & 12 & Never & Computer specialist & Divorced $\times 2$ remarried & 1 \\
3 & F & 39 & 13.5 & Never & Administrative assistant & Divorced remarried & 2 \\
4 & F & 55 & 12 & Never & Secretary & Divorced remarried & 3 \\
5 & M & 46 & 14.5 & Never & Computer consultant & Divorced $\times 2$ remarried & 4 \\
\hline
\end{tabular}


that she did not report odors the previous day; also, no tar, hot or otherwise, was used in the roofing process. She left at 11:00 a.m. Upon arriving home, she spoke on the telephone to her sister, a nurse, who suggested that she had been poisoned. She called the local poison control center, which, based on her story, dispatched an ambulance to her house and alerted Denver Metro Rescue about the office building. She was transported to an emergency department, given oxygen, and told to follow up with an occupational medicine physician. The diagnosis was exposure to fumes.

Records from the poison control center documented that she complained of cough and respiratory irritation. She reported that hundreds of people had been exposed and that many were having symptoms. The poison control center record showed there was a prior visit by case no. 1 to an emergency room in 1993, where she had symptoms of headache, nausea, and general malaise for $24 \mathrm{~h}$. On that occasion she had apparently developed symptoms at work where she reported exposure to sewer gas fumes that had entered her workplace. She was diagnosed as having a flulike syndrome.

On subsequent evaluations, an occupational medicine physician diagnosed RADS and an allergist diagnosed IVCD caused by the building exposures. These physicians determined exposure only from patient recollections, and they did not obtain any empirical exposure level data. A lawsuit ensued and the plaintiff's attorney referred her to another occupational medicine physician who extrapolated her dosage based on her history and symptoms and told her she had been exposed to high levels of toxins. He informed her that she had suffered a brain injury from the building exposure and that she was left hypersensitive to multiple chemicals.

The first occupational medicine physician referred her to a national referral respiratory center. The evaluating physician at the center performed a number of tests including pulmonary function studies and video laryngoscopy and made the diagnosis of IVCD related to the building exposures. She was recruited to participate in a research study on IVCD and was later invited to present her testimonial at a fundraising event for the center.

Case no. 2 This individual did not go to work on 3 November 1999. On 4 November, she worked full day but did not have symptoms until after she left work. She saw the same occupational medicine physician as the other cases, but he did not diagnose her with a respiratory condition and found her to be at maximal-medical improvement (MMI) with no impairment and no work restrictions. Medical records for this time period show a diagnosis of breast cancer and subsequent bilateral mastectomy. Her attorney subsequently referred her to be evaluated by the same physician at a national referral respiratory center where she underwent pulmonary function testing and video laryngoscopy and was diagnosed with IVCD related to building exposures on 4 November 1999.

Case no. 3 During the lunch period on 3 November 1999, she went outside the building with case no. 1, the index case, and another case no. 5, (below) during which they discussed how bad the odors were. She did not report detecting any odors or experiencing symptoms until after returning to the building. On 4 November, she detected odors upon entering the building. While at work, she received a phone call from the index case in which she was told that chemicals used in roofing are toxic and life threatening. She recalled detecting panic in the index case's voice. Fire fighters arrived in a fire truck and ambulance. They were on the phone with the rescue team at the index case's house. The firefighters ordered the building to be evacuated, and offered medical attention to anyone who could stop at the ambulance. She took the option and was administered oxygen, after which she felt better and went home.

She remained symptom free for approximately 6 weeks. On 12 December 1999, she was overwhelmed by uncontrollable cough while dancing at a holiday party. Subsequently, she saw the same occupational medicine physician as the other patients who discharged her at MMI without permanent impairment or need for work restrictions. She was also evaluated by the same allergist who diagnosed IVCD associated with the building exposures. Her attorney subsequently referred her to the same physician at the national referral respiratory center where she was diagnosed with IVCD related to building exposures on 3-4 November 1999. This evaluation notes a panic attack to explain her symptoms during the holiday party. Review of systems identified multisystem symptoms including anxiety, depression, mood swings, and irritability.

Case no. 4 On 3 November, she experienced symptoms in the morning. She heard others talking about feeling ill. She also had symptoms on November 4, but left work early to interview for a new position. She saw the same occupational medicine physician as other plaintiffs who took her off work permanently on the basis of RADS and IVCD. He did not conduct a video laryngoscopy, and the pulmonary function studies he ordered were normal. Nonetheless, this occupational medicine physician noted that she was "intermittently angry, particularly if she perceives any of the questions would suggest that there might be some underlying psychological component to her current symptoms." Additionally, he noted "her multiple neurological complaints and upper respiratory complaints appear to be out of proportion to the amount of exposure she had." And finally, "she reports job dissatisfaction and that she was 
looking for a new job." Her attorney referred her to the second occupational medicine physician, also seen by the index case, who told her the building exposure had left her hypersensitive to multiple chemicals. The medical records corroborated her account. She was also referred to the same physician at the national referral respiratory center but did not follow through on the recommended evaluation that included additional pulmonary function testing and video laryngoscopy.

Case \# $5 \mathrm{He}$ did not report symptoms on 3 November until after the lunch period meeting with two other cases when they discussed the odors. He did not report to work on 4 November. On 8 November, he went to an emergency department for evaluation and was referred to the same occupational medicine physician who saw the other patients. He was diagnosed with RADS and referred to the same physician at the national referral respiratory center who performed pulmonary function studies and video laryngoscopy and diagnosed him as having IVCD related to building exposures on 3-4 November 1999. The patient was recruited to participate in a research study on IVCD. He could not return to the building because of alleged reactions to multiple chemical exposures. He dated his belief that all his ailments were due to the exposures in the building to a phone call from the index case on 4 November, when he was out sick at home. She told him that the building had been evacuated and that several people had been sent to the hospital because of the toxic exposure to fumes.

\section{Signs and Symptoms}

Symptoms reported by the five cases during the initial event and 3 years later are presented in Table 2. The collective diverse, multisystem complaints during the alleged expo-

Table 2 Symptoms (frequency) for five cases

\begin{tabular}{ll} 
During alleged exposure, Nov. 3-4, 1999 & 3 years later \\
\hline Headache (5) & Cognitive dysfunction (5) \\
Nausea (5) & Fatigue (5) \\
Vomiting (3) & Headache (4) \\
Dizziness (2) & Cough (3) \\
Cough (2) & Dizziness (2) \\
Short of breath (2) & Syncope (1) \\
Confusion, disorientation (2) & Paresthesia (1) \\
Difficulty concentrating (1) & Chest tightness (1) \\
Dysphonia (1) & Nausea (1) \\
Chest tightness (1) & Vomiting (1) \\
& Hoarseness (1) \\
\hline
\end{tabular}

sure and 3 years later are not specific for injury to the airway presenting as IVCD or RADS. The diverse symptoms were unsupported by objective findings. The symptoms at onset are consistent with anxiety and panic $[16,17]$.

Physical examinations of each of these plaintiffs by one of the authors (LR), specifically relevant to all of their building associated complaints, were entirely normal. Complete pulmonary function tests (spirometry, plethysmographic lung volumes, and diffusing capacity) were either uninterpretable due to poor effort and cooperation, or were entirely normal. Methacholine challenge tests, performed according to the methods of the American Thoracic Society Consensus Statement [18], were either uninterpretable due to poor effort and cooperation, or were entirely negative for airways hyperreactivity. Review of videotapes obtained on the four patients during laryngoscopic examination by one of the authors $(\mathrm{KC})$ failed to support the diagnosis of VCD [19, 20].

\section{Air Sampling and Exposure Modeling}

Initial investigation of the roof repair by the responding fire department observed an open container of primer used to seal the roofing membrane in close proximity to the air intake on the roof. The material safety data sheet for the primer listed toluene as the primary solvent at a concentration of 90\%. On 4 November 1999 at 3:00 p.m., members of the rescue services department took air sampling measurements inside the incident building indicative of total VOCs including toluene, with a hand-held, real-time VOC monitor and reported a measurement of zero to $1 \mathrm{ppm}$. For purposes of comparison, background air measurements taken 3 years later with another VOC monitor inside a nearby building and outside in a parking lot ranged from zero to $2.7 \mathrm{ppm}$. The building owner contracted an industrial hygiene firm and on 5 November, monitoring was conducted at 17 sites in the building, and on 8 November, a total of 27 sites were monitored. No "unique" odors were detected during the sampling and walk-through of the building on these dates. All measurements showed 0 ppm of VOCs, indicating only background concentrations.

Toluene was identified to be the primary constituent of the three most used chemical roofing solvents and glues, and was used as the contaminant of concern for modeling. Toluene odor threshold is $2.5 \mathrm{ppm}$ [21], well below the OSHA Permissible Exposure limit of 200 ppm; the 20 ppm limit published by the American Conference of Governmental Industrial Hygienists (ACGIH Threshold Limit value (TLV); and the 50 ppm EPRG-1 limit established by the American Industrial Hygiene Association (AIHA Emergency Planning Response Guideline). The AIHA defines the ERPG-1 as the 
maximum airborne concentration below which it is believed nearly all individuals could be exposed for up to $1 \mathrm{~h}$ without experiencing more than mild, transient adverse health effects or without perceiving a clearly defined objectionable odor. AIHA also has established an ERPG-2 of 300 ppm, which is the maximum airborne concentration below which it is believed nearly all individuals could be exposed for up to $1 \mathrm{~h}$ without experiencing or developing irreversible or other serious health effects or symptoms that could impair an individual's ability to take protective action. The reported exposure time of the plaintiffs on 3 November 1999 was estimated at $6.5 \mathrm{~h}$; and on 4 November 1999, at $4.5 \mathrm{~h}$, for a maximum possible total exposure time of $11 \mathrm{~h}$. Each of the plaintiffs worked in the office/cubicle area in the central part of the third floor along with dozens of other workers who did not file complaints in regard to the subject incident.

Toluene has the lowest exposure limits among the VOCs identified as product constituents. Fixed area air samplers during a roofing membrane application at a similar site measured VOCs at $11 \mathrm{ppm}$. Among these VOCs, toluene was the most prevalent of the air contaminants detected, and was measured at $8.6 \mathrm{ppm}$. Air concentrations of acetone, heptane, isopropanol, and xylenes, other chemicals in the roofing glues and primers, were determined to be individually below 1 ppm. These air contaminant samples were collected with battery-powered, industrial hygiene personal air sampling pumps drawing air through activated charcoal tube media. Sample results were determined by a laboratory accredited in industrial hygiene analysis. For toluene, the lower limit of detection for this method is a nominal $0.01 \mathrm{ppm}$.

Measurements of VOCs emissions were recorded during short, discrete work tasks, e.g., applying primer to roofing membrane seams at the similar site. These measurements were collected with a VOC monitor, calibrated on the day of use, and were used to develop models of contaminated volumes of air entering the office building in 1999. These models estimated that the VOC concentrations ranged up to 15 ppm.

A theoretical, worst-case mathematical model, assuming a spill at Denver's altitude and complete evaporation inside the building of 1 gal of roofing membrane primer made up of $100 \%$ toluene, revealed an upper limit of $41 \mathrm{ppm}$. This modeling was performed to determine the absolute upper bound of solvent vapors that could have occurred inside the building during the November 1999 incident. One gallon is the approximate amount of primer to complete the daily sealing of overlapping membrane seams on the roof at the office building. This scenario would not likely occur since: (1) overly conservative assumptions were used in development of the model; (2) wind conditions typically found atop a building of this height would result in the majority of toluene emissions from the process being diluted or blown away from the fresh air intake of the building; and (3) the primer was applied over an entire nominal 8-h work shift and not instantaneously as would occur in a spill of the contents of the entire primer container. While this is not a realistic depiction of the events of 3-4 November 1999, this worst-case model serves as a useful basis of assessment of possible dose and toxicity.

Tracer gas testing using sulfur hexafluoride was used to simulate the chemical exposure scenario that could have occurred during the roofing membrane application. Sulfur hexafluoride was injected into the fresh air intake of the building's HVAC system late at night when all building occupants were gone. Conservative assumptions were applied, as the injection rate for the tracer gas was based on the highest anticipated toluene emission rate calculated based on a day's usage of the primer of concern. During tracer gas testing in 2003, the building's HVAC system was configured to operate under conditions similar to those on 3-4 November 1999. A quantitative gas analyzer with a photo-acoustic infrared detector, capable of sub ppm measurements, was used to measure the tracer gas. Measurements recorded on the 3 rd floor where the impacted employees worked revealed that toluene concentrations would not have exceeded $20 \mathrm{ppm}$.

The design forced ventilation of the building is 60,000 cubic feet per minute (cfm). On 3-4 November 1999, air handlers were operating at approximately $30 \%$ of maximum design flow, or $18,000 \mathrm{cfm}$. With a building volume of 820,000 cubic feet, the calculated complete air exchange time for the building is $14 \mathrm{~min}$ (by design) to $45 \mathrm{~min}$ (per subject incident estimated flow). However, air exchanges would occur more frequently than these calculated rates because of openings in doorways, stairwells, elevator shafts, utility chases, crack in walls, and other openings present in all buildings. Modeling based on toluene levels determined from the fixed monitors and the VOC monitor were based on $30 \%$ of design ventilation rates. The tracer gas measurements are real-time, and as such, incorporate building air exchanges from the actual forced ventilation and the natural ventilation occurring in the building at the time of the measurements. The spill "worst-case" scenario assumed static building conditions with no air exchanges.

\section{Dose Estimate}

A relative estimate of dose experienced by persons involved in the subject incident can be shown by computing ppm-hours for the incident versus an exposure limit. The ACGIH TLV for an 8-h work shift is 20 ppm. By ACGIH's definition, this time-weighted average TLV is the air concentration to which it is believed that nearly all workers may be repeatedly exposed, day after day, for a 
working lifetime without adverse effect [22]. For a 40-h week, the imputed dose should not exceed $800 \mathrm{ppm}-\mathrm{h}(20 \mathrm{ppm} \times$ $40 \mathrm{~h}$ ). The data collected during this study suggest that the incident's five cases had a dose up to 220 ppm-hours $(20 \mathrm{ppm} \times 11 \mathrm{~h})$, which is $28 \%$ of the imputed weekly occupational dose. This dose is the maximum possible, since some of the cases did not go to work for the full 2 days, as detailed in their case histories, above. At this dose, serious, long-lasting health effects are not expected.

\section{Psychological Testing}

The results of the psychological testing are presented in Table 3. Somatization and obsessive-compulsiveness were the most frequent SCL-90R categories of current distress. The MMPI- 2 validity scales showed that three of the cases adopted a defensive approach. T scores $>64$ on the MMPI-2 validity scales $\mathrm{L}, \mathrm{K}$, and $\mathrm{S}$ reflect defensiveness characterized by denial of common human frailties, denial of psychological problems, and exaggerated virtue. None of the cases over-reported infrequent psychiatric symptoms, as would be indicated by an elevated F scale. On the MMPI-2 clinical scales 1 (Hs) and 3 (Hy), four cases showed elevations with $\mathrm{T}$ score $>64$ on both and one showed elevation on scale 3 only. Two cases demonstrated poor effort as measured by the CARB and WMT tests. In all cases, the MCMI-II identified traits associated with various personality disorders, which were confirmed by interview.

\section{Medical and Stress History}

The medical, mental health, and stress histories obtained during the psychological evaluation are presented in Table 4. The mental health histories are remarkable for multiple risk factors for emotional distress and lack of treatment.

\section{Discussion}

To our knowledge, this is the first study of MPI in which rigorous and comprehensive evaluation of cases' medical and psychological conditions were conducted in addition to industrial hygiene assessment. As previously noted, $[1,7$, 23] social interactions among the patients contributed to the creation of a belief of harm and disability and initiated this incident of MPI. A purely sociological theoretical perspective of MPI suggests that interpersonal sociological phenomenon can affect anyone at random. This study demonstrated that the patients had psychological personality traits that predisposed them to create or be affected by the social aspects of MPI.

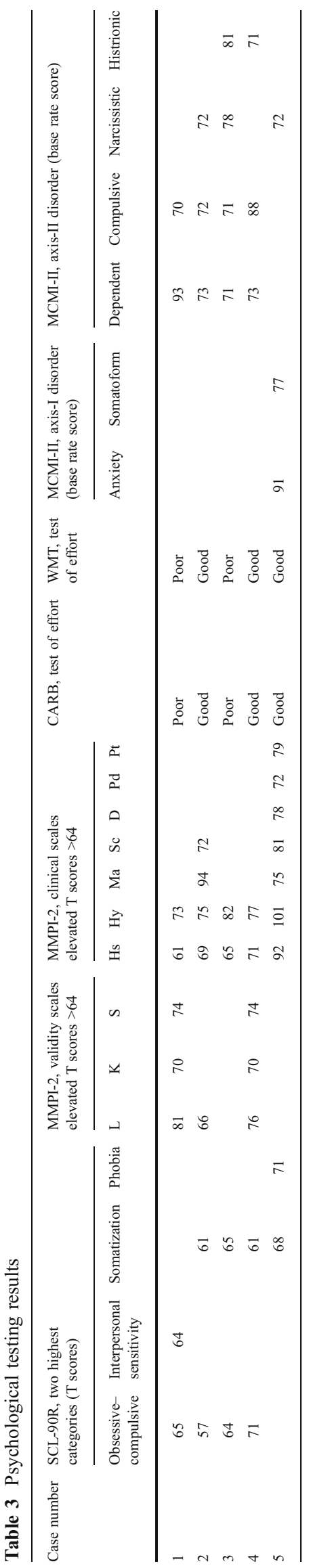


Table 4 Premorbid medical, mental health, and stress history

\begin{tabular}{|c|c|c|c|}
\hline Case no. & Medical history & Mental health history & Stress history \\
\hline 1 & $\begin{array}{l}\text { Meningioma surgery, 1988; } \\
\text { laminectomy, } 1980\end{array}$ & $\begin{array}{l}\text { Grief counseling, 1987; denies } \\
\text { psychotropic medications }\end{array}$ & $\begin{array}{l}\text { Death of baby; second child with life } \\
\text { threatening heart condition; and } 1993 \text { reports } \\
\text { hydrogen sulfide exposure to poison control } \\
\text { center }\end{array}$ \\
\hline 2 & $\begin{array}{l}\text { Irritable bowel syndrome, } 1990 \text {; } \\
\text { bilateral mastectomy, 2000; } \\
\text { medically unsubstantiated } \\
\text { "stroke", } 2002\end{array}$ & $\begin{array}{l}\text { Counseling during spousal abuse and } \\
\text { denies psychotropic medications }\end{array}$ & $\begin{array}{l}\text { Spousal abuse, sexual, physical, and } \\
\text { psychological, 1993, with posttraumatic } \\
\text { stress disorder sequelae; father dies, 1999; } \\
\text { breast cancer, 1999; and "stroke" after } \\
\text { argument with son, } 2002\end{array}$ \\
\hline 3 & $\begin{array}{l}\text { Motor vehicle accident resulting } \\
\text { in lower back injury, } 1999\end{array}$ & $\begin{array}{l}\text { Marriage counseling, first marriage } \\
\text { and denies psychotropic mediations }\end{array}$ & $\begin{array}{l}\text { Father neglecting; mother mentally unstable; } \\
\text { low self esteem; dislike of men, detested 1st } \\
\text { husband; 2nd husband's daughter disrupted } \\
\text { family, left her feeling resentful and angry; } \\
\text { and she blamed her son's oppositional } \\
\text { defiant disorder on her own anger during } \\
\text { his pregnancy }\end{array}$ \\
\hline 4 & Hypertension & $\begin{array}{l}\text { Psychological evaluation, 2001; denies } \\
\text { psychotherapy; and medications: } \\
\text { Zoloft and Celexa for panic }\end{array}$ & $\begin{array}{l}\text { 1st husband unfaithful; divorced, } 3 \text { sons went } \\
\text { with father; 2nd husband physically abusive; } \\
\text { separation with 2nd husband while 1st wife; } \\
\text { later she reconciled with 2nd husband }\end{array}$ \\
\hline 5 & Hypertension and knee surgery & $\begin{array}{l}\text { Denies psychotherapy; denies psychotropic } \\
\text { mediations and pre-incident } \\
\text { psychological records showed a } \\
\text { diagnosis of panic disorder with } \\
\text { agoraphobia }\end{array}$ & $\begin{array}{l}\text { 2nd wife drug addict; child conceived out of } \\
\text { wedlock, he paid alimony; } 2 \text { nd child out of } \\
\text { wedlock, married the mother } 11 \text { years later; } \\
\text { large debt from lost legal case for failure to } \\
\text { pay contractor; and delinquent IRS taxes } \\
\text { with penalties }\end{array}$ \\
\hline
\end{tabular}

In the standard psychiatric nomenclature, MPI is subsumed under the general heading of somatoform disorder, subcategorized as conversion disorder [24]. This classification specifies that certain factors be observed in the index case(s) such as medically unexplained symptoms associated with somatization, medical seeking behavior to confirm a physical illness and maintain the sick role, denial of a psychological etiology, and alternative psychological factors to explain the ailments. These factors were identified not only in the index case, but in all cases. The initial symptoms (e.g., headaches, nausea and vomiting, shortness of breath) reflect symptoms of anxiety or panic induced by the perception of hazardous exposure [16]. The chronic multiorgan system complaints reflect beliefs molded by the prevailing fashionable somatic syndrome of IEI [25] presenting as "sick building syndrome." Professional organizations have rejected IEI as a medical diagnosis [26, 27].

This is the first MPI study to demonstrate the potential iatrogenic role physicians play in reinforcing beliefs of a toxic induced illness by "diagnosing" unsubstantiated or pseudo-disorders that are semantic variations of IEI. In addition to the harm associated with false beliefs of a toxic illness, four cases in this series received medications to treat pseudo-disorders. Some medications, particularly systemic oral corticosteroids, have associated risks to which patients were needlessly exposed.

There are several respiratory conditions that have been attributed to chemical intolerance that are without scientific foundation including IVCD. The single IVCD study published to date presented IVCD as a separate and distinct entity from VCD. It was designed as a retrospective chart review, which identified 11 patients who self-reported symptom onset temporally related to chemical exposure [9]. VCD is a well-described disorder with a characteristic clinical presentation that includes shortness of breath with audible wheeze and established laryngoscopic diagnostic criteria reliant upon proper laryngoscopic examination [19, 20]. The preponderance of the literature recognizes a psychologic etiology for these paroxysms of glottic obstruction due to paradoxical vocal cord adduction. Emotional distress, somatization, and poor ability to cope with stress are identified to be prevalent. Premorbid psychopathology including personality disorders play a significant role in the etiology of VCD [28], which is most often diagnosed as a somatoform disorder, specifically conversion disorder [29]. Evidence of organic disease, disorder or injury are absent and the psychologic nature can be demonstrated by a variety of methods including response to administration and withdrawal of helium, 
response to placebo, resolution when the patient is distracted, respiratory maneuvers, and blinded provocation testing with self-reported triggers $[19,30]$.

Even though each of the cases in this report was diagnosed as having IVCD, none of the cases actually had VCD. Case no. 3 had laryngoscopic findings that have been previously noted when conscious efforts are made to produce laryngeal obstruction [19]. This individual was also one of two cases who met criteria for malingering by showing poor effort on cognitive performance tests of effort indicating exaggeration or misrepresentation of symptoms of neurocognitive dysfunction [31]. However, even if all five did have VCD, a psychological etiology for presenting respiratory symptoms predominates over an unsubstantiated theory of glottic injury with chronic respiratory impairment triggered by exposures to low-level multiple chemicals [30].

The MMPI-2 score elevations are typical in personal injury plaintiffs and represent the self-serving exaggeration of health difficulties $[32,33]$. Over time, these misrepresentations interacted with social and iatrogenic factors to strengthen the conviction of toxic harm. By attributing medically unexplained symptoms to the environment, unresolved psychological conflicts stemming from premorbid distressing life events that the patient cannot cope with are projected external to the self.

In conclusion, we have presented a unique study in which rigorous industrial hygiene assessment demonstrated that no hazardous exposure had occurred, psychological assessment identified long-standing psychopathology that could explain the reporting of medically unsubstantiated symptoms, and medical evaluation exposed unwarranted "diagnoses" of pseudo-IVCD and pseudo-RADS. Psychological etiology should be considered in cases of building-related illness and "sick building syndrome," especially when there is sudden onset of symptoms characteristic of MPI, which progress to chronic multiorgan system complaints attributed to an ever spreading array of environmental agents, a phenomenon without scientific basis for toxic causation [34].

Conflicts of Interest Statement The authors were retained by counsel for the defendants to conduct independent evaluations in this litigation for which they were reimbursed for their time. No financial support was received for preparation of this paper.

\section{References}

1. Sirois F (1982) Perspective on epidemic hysteria. In: Colligan M, Pennebaker J, Murphy P (eds) Mass psychogenic illness: a social psychological perspective. Lawrence Erlbaum, Hillsdale, pp 217236

2. Boss LP (1997) Epidemic hysteria: a review of the published literature. Epidemiol Rev 19:232-243
3. Bartholomew RE, Wessely S (2002) Protean nature of mass psychogenic illness. Br J Psychiatry 180:300-306

4. Shorter E (1992) From paralysis to fatigue: a history of psychosomatic illness in the modern era. Free Press, New York

5. Shusterman D (2002) Review of the upper airway, including olfaction, as mediator of symptoms. Env Health Persp 110(Suppl 4):649-653

6. Hodgson M (2002) Indoor environmental exposures and symptoms. Env Health Persp 110(Suppl 4):663-667

7. Jones TF, Craig AS, Hoy D et al (2000) Mass psychogenic illness attributed to toxic exposure at a high school. N Engl J Med 342:96-100

8. Brooks SM, Weiss MA, Bernstein IL (1985) Reactive airways dysfunction syndrome (RADS): persistent asthma syndrome after high level irritant exposure. Chest 88:376-384

9. Perkner JJ, Fennelly KP, Balkissoon R et al (1998) Irritant-associated vocal cord dysfunction. J Occup Environ Med 40:136-143

10. International Programme on Chemical Safety and World Health Organization (1996) Conclusions and recommendations of a workshop on multiple chemical sensitivities (MCS). Reg Toxicol Pharmacol 24(1):S188-S189

11. Derogatis LR (1983) Administration, scoring and procedures manual, II. Clinical Psychometric Research, Towson

12. Butcher JN, Dahlstrom WG, Graham JR et al (1989) MMPI-2 manual for administration and scoring. University of Minnesota Press, Minneapolis

13. Millon T (1987) Manual for the MCMI-II, 2nd edn. National Computer Systems, Inc., Minneapolis

14. Allen LM, Conder RL, Green P et al (1998) CARB'97: computerized assessment of response bias. CogniSyst, Inc, Durham

15. Green P, Allen LM, Astner K (1996) The Word Memory Test: a user's guide to the oral and computer-administered forms, US version 1.1. CogniSyst, Inc, Durham

16. Leznoff A (1997) Provocation challenges in patients with multiple chemical sensitivity. J Allergy Clin Immunol 99:438-442

17. Homnick DN, Pratt HD (2000) Respiratory diseases with a psychosomatic component in adolescents. Adolsc Med 11:547565

18. American Thoracic Society (2000) Guidelines for methacholine and exercise challenge testing-1999. Am J Respir Crit Care Med 161:309-329

19. Christopher KL, Wood RP, Eckert RC, Blager FB, Raney RA, Souhrada JF (1983) Vocal-cord dysfunction presenting as asthma. N Engl J Med 308:1566-1570

20. Wood RP, Milgrom H (1996) Vocal cord dysfunction. J Allergy Clin Immunol 98:481-485

21. American Conference of Governmental Industrial Hygienists, Inc (2001) Documentation of the threshold limit values and biological exposure indices: toluene. ACGIH, Cincinnati

22. American Conference of Governmental Industrial Hygienists, Inc (2006) TLV's and BEIs (Registered Trademarks) Based on the documentation of the threshold limit values for chemical subtances and physical agents and biological exposure indices. ACGIH, Cincinnati

23. Colligan MJ, Pennebaker JW, Murphy LR (eds) (1982) Mass psychogenic illness: a social psychological perspective. Lawrence Erlbaum, Hillsdale

24. American Psychiatric Association (1994) Diagnostic and statistical manual of mental disorders (DSM-IV), 4th edn. American Psychiatric Press, Washington

25. Barsky AJ, Borus JF (1999) Functional somatic syndromes. Ann Int Med 130:910-921

26. American Academy of Allergy Asthma and Immunology (1999) Board of directors position statement: idiopathic environmental intolerances. J Allergy Clin Immunol 103:36-40 
27. American College of Occupational and Environmental Medicine. Multiple chemical sensitivities: idiopathic environmental intolerance. Position Statement. ACOEM Report 1999, 1-3 June

28. Freedman MR, Rosenberg SJ, Schmaling KB (1991) Childhood sexual abuse in patients with paradoxical vocal cord dysfunction. J Nervous Mental Disease 179:295-298

29. Lacy TJ, McManis SE (1994) Psychogenic stridor. Gen Hosp Psychiatry 16:213-223

30. Selner JC, Staudenmayer H, Koepke JW et al (1987) Vocal cord dysfunction: the importance of psychologic factors and provocation challenge testing. J Allergy Clin Immunol 79:726-733
31. Slick DJ, Sherman EMS, Iverson GL (1999) Diagnostic criteria for malingering neurocognitive dysfunction: proposed standards for clinical practice and research. Clin Neuropsychology 13:545-561

32. Lees-Haley PR (1997) MMPI-2 base rates for 492 personal injury plaintiffs: implications and challenges for forensic assessment. J Clin Psychol 53:745-755

33. Lanyon RI (2001) Dimensions of self-serving misrepresentation in forensic assessment. J Pers Assess 76:169-179

34. Staudenmayer H, Binkley KE, Leznoff A et al (2003) Idiopathic environmental intolerance (IEI) part I: a causation analysis applying bradford hill's criteria to the toxicogenic theory. Toxicol Rev 22:235-246 